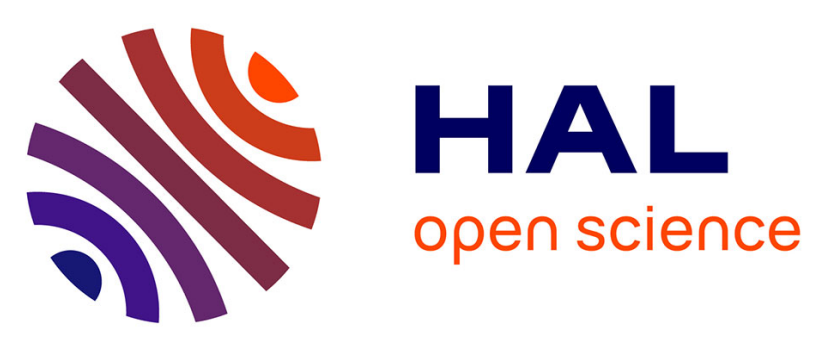

\title{
Assessment of HBEC-5i endothelial cell line cultivated in astrocyte conditioned medium as a human blood-brain barrier model for ABC drug transport studies
}

Clémentine Puech, Sophie Hodin, Valérie Forest, Zhiguo He, Patrick

Mismetti, Xavier Delavenne, Nathalie Perek

\section{To cite this version:}

Clémentine Puech, Sophie Hodin, Valérie Forest, Zhiguo He, Patrick Mismetti, et al.. Assessment of HBEC-5i endothelial cell line cultivated in astrocyte conditioned medium as a human blood-brain barrier model for ABC drug transport studies. International Journal of Pharmaceutics, 2018, 551 (1-2), pp.281-289. 10.1016/j.ijpharm.2018.09.040 . hal-01892564

\section{HAL Id: hal-01892564 \\ https://hal.science/hal-01892564}

Submitted on 10 Oct 2018

HAL is a multi-disciplinary open access archive for the deposit and dissemination of scientific research documents, whether they are published or not. The documents may come from teaching and research institutions in France or abroad, or from public or private research centers.
L'archive ouverte pluridisciplinaire HAL, est destinée au dépôt et à la diffusion de documents scientifiques de niveau recherche, publiés ou non, émanant des établissements d'enseignement et de recherche français ou étrangers, des laboratoires publics ou privés. 
Assessment of HBEC-5i endothelial cell line cultivated in astrocyte conditioned medium as a human blood-brain barrier model for $\mathrm{ABC}$ drug transport studies

Clémentine Puech ${ }^{\mathrm{a}, \mathrm{b} *}$, Sophie Hodin ${ }^{\mathrm{a}, \mathrm{b}}$, Valérie Forest ${ }^{\mathrm{c}}$, Zhiguo He $\mathrm{He}^{\mathrm{b}, \mathrm{d}}$, Patrick Mismetti $^{\mathrm{a}, \mathrm{b}, \mathrm{e}}$, Xavier Delavenne ${ }^{\mathrm{a}, \mathrm{b}, \mathrm{f}}$, Nathalie Perek ${ }^{\mathrm{a}, \mathrm{b}}$.

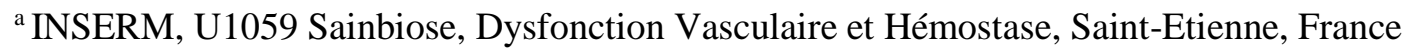

${ }^{\mathrm{b}}$ Université de Lyon, Saint-Etienne, F-42023, France

${ }^{\mathrm{c}}$ Mines Saint-Etienne, Univ Lyon, Univ Jean Monnet, INSERM, U 1059 Sainbiose, Centre CIS, F42023 Saint-Etienne, France

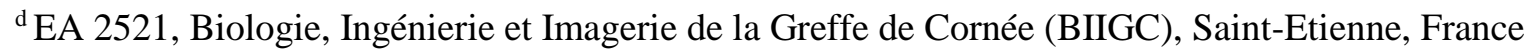

${ }^{\text {e }}$ Unité de Recherche Clinique Innovation et Pharmacologie, CHU de Saint-Etienne, F-42055 Saint Etienne, France

${ }_{\mathrm{f}}^{\mathrm{f}}$ Laboratoire de Pharmacologie Toxicologie, CHU Saint-Etienne, F-42055 Saint-Etienne, France

* Corresponding author: Clémentine Puech e-mail: c.puech@univ-st-etienne.fr

Postal address: 10 rue de la Marandière BP 80019, Faculté de Médecine, 42270 Saint-Priesten-Jarez, France

Tel: (+33)4.77.42.14.43 


\section{Abstract:}

Endothelial cells are main components of the Blood-Brain Barrier (BBB) and form a tight monolayer that regulates the passage of molecules, with the ATP-Binding Cassette (ABC) transporters efflux pumps. We have developed a human in vitro model of HBEC-5i endothelial cells cultivated alone or with human astrocytes conditioned medium on insert. HBEC-5i cells showed a tight monolayer within 14 days, expressing ZO-1 and claudin 5, a low apparent permeability to small molecules, with a TEER stability during five days. The P-gp, BCRP, MRPs transporters were well expressed and functional. Accumulation and efflux ratio measurement with different $\mathrm{ABC}$ transporters substrates (Rhodamine 123, BCECF AM, Hoechst 33342) and inhibitors (verapamil, Ko143, probenecid and cyclosporin A) were conducted. At barrier level, the functionality of $\mathrm{ABC}$ transporters was three-fold enhanced in astrocyte conditioned medium. We validated our model by the transport of pharmacological substrates: caffeine, rivaroxaban, and methotrexate. The rivaroxaban and methotrexate were released with an efflux ratio $>3$ and were decreased by more than half with inhibitors. HBEC5i model could be used as relevant tool in preclinical studies for assessing the permeability of therapeutic molecules to cross human BBB.

Keys words: Blood-Brain Barrier; ABC transporters, efflux ratio, human in vitro models, astrocyte conditioned medium. 


\section{Introduction}

The Blood-Brain Barrier (BBB) is a protective barrier between the blood and the Central Nervous System (CNS)(Eigenmann et al., 2013). The BBB is composed of endothelial cells, pericytes, astrocytes, and neurons, the whole forming the neurovascular unit. Endothelial cells are the main components of the BBB because they restrict and control the passage of molecules inside and outside the brain. Barrier property of endothelial cells is mainly provided by tight junction proteins (TJs), and ATP Binding Cassette (ABC) transporters (Abbott, 2013).

$\mathrm{ABC}$ transporters are a large family of transporters, the most involved in $\mathrm{BBB}$ are $\mathrm{P}-$ glycoprotein protein (P-gp), Breast Cancer Resistance Protein (BCRP) and Multidrug Resistance associated Proteins (MRPs). They are responsible for active efflux of therapeutic agents. It has been estimated that less than $2 \%$ of small molecule drugs are able to cross the BBB (Eigenmann et al., 2013; Pardridge, 2005; Ronaldson and Davis, 2015). The prediction of drug passage through $\mathrm{BBB}$ and involvement of $\mathrm{ABC}$ transporters represent major issues in drug development.

The evaluation of drug transport through the $\mathrm{BBB}$ mediated by $\mathrm{ABC}$ transporters requires an in vitro human barrier model which must fulfil different criteria to be considered as a functional and adequate model for drug research development. These criteria include: the permeability to several compounds, the presence of TJs, and the expression of functional $\mathrm{ABC}$ transporters with bidirectional studies and efflux ratio (ER) measurement (Food and drug administration, 2012; Puech et al., 2018). This latter is possible only with a monolayer of endothelial cells cultivated on a filter. Currently, no standard human BBB model to study interactions of drugs with $\mathrm{ABC}$ transporters correspond entirely to these criteria. There is a need to develop models that form an adequate monolayer on filter.

Several human cell lines were investigated in the literature i.e. TY08 (Sano et al., 2010), TY10 (Eigenmann et al., 2013), HCEC (Muruganandam et al., 1997), HMEC-1 (Ades et al., 1992), BB19 (Prudhomme et al., 1996), HBMEC (Eigenmann et al., 2013), hCMEC/D3 
(Weksler et al., 2005). In the literature, the major human endothelial cell lines that have been well characterized are human Cerebro Microvascular Endothelial Cells (hCMEC/D3) (Weksler et al., 2013) and Human Brain Microvascular Endothelial Cells (hBMEC)(Eigenmann et al., 2016b). However, these models are grown in a medium containing a cocktail of adjuvants (Eigenmann et al., 2016b; Kulczar et al., 2017; Weksler et al., 2013) and it is known that adjuvants have an impact on the expression of ABC transporters (Eisenblätter and Galla, 2002; Maines et al., 2005; von Wedel-Parlow et al., 2009).

In this work, we have chosen another human brain endothelial cell line: HBEC-5i (Jiang et al., 2017) that can grow in monolayer on inserts without adjuvants. Furthermore, this cell line was cultivated in presence of human astrocyte medium as the barrier properties are mostly induced and maintained by the close links between brain endothelial cells and astrocytes (Abbott et al., 2006; Janzer and Raff, 1987). In the literature, it has been described that the soluble factor released by the astrocytes can induce modifications in the expression of functionality of the P-gp (Abbott et al., 2006; Berezowski et al., 2012; Dohgu et al., 2005). We have compared the monoculture in standard medium with a model with astrocyte conditioned medium. ABC transporters functionality was evaluated with accumulation and bidirectional studies as well as ER (efflux ratio) parameter with fluorescent selective substrates and inhibitors. HBEC-5i monolayer presents an adequate BBB model that can be routinely used to evaluate drug interactions with $\mathrm{ABC}$ transporters.

\section{Material and methods}

\subsection{Chemicals and reagents}

Cell culture inserts for 24-well plates (High Density of pore, $0.4 \mu \mathrm{m}$ pore diameter size, translucent poly-terephthalate ethylene (PET) membrane were purchased from BectonDickinson (Franklin Lakes, USA). EVOM ${ }^{\circledR}$ voltohmmeter system was purchased from World 
Precision Instruments with STX-2 electrode (Sarasota, USA). Twenty-five culture flasks and companion plates were purchased from Dominique Dutscher (Strasbourg, France).

Rabbit polyclonal anti-vascular endothelial cadherin (VE-cadherin-ab33168), rabbit polyclonal anti-Zonula Occulens-1 (ZO-1ab59720) and rabbit polyclonal anti-P-gp (ab129450) antibodies for immunofluorescence (IF) and ELISA were purchased from Abcam (Cambridge, United Kingdom). Rabbit polyclonal anti-BCRP(sc-25822), rabbit polyclonal anti-MRP-1(sc-13960), rabbit polyclonal anti-MRP-2 (sc-20766), and rabbit polyclonal anti-claudin 5 (sc-28670) antibodies for IF or ELISA and mouse monoclonal anti-rabbit IgG-biotin for cell ELISA were purchased from Santa Cruz Biotechnology (Dallas, Texas, U.S.A). Hoechst 33342, rhodamine 123 and secondary antibodies for IF: goat anti-rabbit antibodies (A-21127Alexa Fluor ${ }^{\mathrm{TM}} 555$ ), 4', 6-diamidino-2-phenylindole (DAPI) for microscopy and Coomassie plus ${ }^{\mathrm{TM}}$ protein assay and superFrost ${ }^{\mathrm{TM}}$ microscope slide were obtained from Thermo Fischer Scientific (Waltham, USA).

Astrocyte Medium (AM), Astrocyte Growth Supplement (AGS), Fetal Bovine Serum (FBS) and penicillin-streptomycin solution for astrocytes were purchased from CliniSciences (Nanterre, France). Rivaroxaban, $\left[{ }^{13} \mathrm{C}_{6}\right]$-rivaroxaban, caffeine, methotrexate and $\left[{ }^{13} \mathrm{C},{ }^{2} \mathrm{H}_{3}\right]$ methotrexate were obtained from Alsachim (Illkirch, France). Caffeine-D9 was purchased from LGC standard (Molsheim, France). Normal growth supplement (NGS) was obtained from Millipore (Burlington, USA).

All other reagents were purchased from Sigma-Aldrich (St Quentin Fallavier, France).

\subsection{In vitro BBB model}

\subsubsection{Cell culture}

The model was composed of HBEC-5i endothelial cells (from ATCC-Manassas, VA, USA) and the Human Astrocyte cell line (HA from ScienCell Research Laboratories, Carlsbad, CA, USA). 
Initially, HBEC-5i were cultured in Dulbecco's Modified Eagle Medium: nutrient Mixture F-12 (DMEM-F12 HAM), supplemented with 10\% FBS, $40 \mu \mathrm{g} / \mathrm{mL}$ endothelial cell growth supplement (ECGS), and 1\% antibiotic antimycotic solution and HA were grown in AM Medium containing 2\% FBS, $1 \%$ AGS and $1 \%$ penicillin-streptomycin solution according to manufacturer's instructions. For the drug transport studies, the two cell lines were cultured in a DMEM-F12 HAM medium without ECGS.

A monoculture model with endothelial cell line was performed, and cooperation with astrocytes was also evaluated with astrocytes conditioned medium. Endothelial cells were seeded at a density of $2 \times 10^{5}$ cells/wells on translucent PET culture inserts with a $0.33 \mathrm{~cm}^{2}$ growth area and high-density of $0.4 \mu \mathrm{m}$ pore size. For the conditioned medium, a monoculture of HBEC-5i was cultivated with astrocyte conditioned medium at the basolateral side of the filter. For each condition, the medium was renewed every two days.

\subsubsection{Barrier properties}

The apparent permeability $\left(\mathrm{P}_{\mathrm{app}}\right)$ was assessed using two hydrophilic fluorescent molecules: sodium fluorescein $(\mathrm{Na}-\mathrm{Fl} \mathrm{MW}=376 \mathrm{Da})$ or fluorescein isothyocyanate Dextran (Dextran-FITC MW= $4 \mathrm{kDa}$ ). Hank's balanced salt sodium (HBSS) transporter buffer supplemented with $10 \mathrm{mM}$ HEPES containing $10 \mu \mathrm{g} / \mathrm{mL}$ of $\mathrm{Na}-\mathrm{Fl}$ or dextran-FITC was loaded onto the apical side of the insert and incubated at $37^{\circ} \mathrm{C}$ for 1 hour. Fluorescence was measured with a fluorescence spectrophotometer (Fluoroskan Ascent ${ }^{\mathrm{TM}}$, Thermo Fischer scientific, France) with $485 \mathrm{~nm}$ excitation and $530 \mathrm{~nm}$ emission wavelengths. The permeability for all molecules was computed using the following formula:

$$
P_{a p p}=\frac{V_{r}}{C_{0}} \times \frac{1}{S} \times \frac{C_{1}}{t}(\text { Formula } 1)
$$

Where $\mathrm{P}_{\text {app }}$ is the apparent permeability, $\mathrm{Vr}$ is the volume of medium in the receiver chamber, $\mathrm{C}_{0}$ is the concentration of fluorescent compound in the donor chamber at $\mathrm{T}_{0}, \mathrm{~S}$ is the surface area of the monolayer, $\mathrm{C}_{1}$ is the concentration of fluorescent molecule in the receiver chamber 
after 1 hour of incubation and $\mathrm{t}$ is the incubation time. The results are expressed in $\mathrm{cm}^{-1} \mathrm{~s}^{-1}$ (Hodin et al., 2017; Puech et al., 2018). The evaluation of a passage of pharmacological hydrophilic molecule, the caffeine (MW=194 Da) was performed in the same way. The quantification was carried out in liquid chromatography-mass spectrometry (LC-MS: Waters, Saint-Quentin-enYvelines, France).

Trans-Endothelial Electrical Resistance (TEER) was recorded using an EVOM $^{\circledR}$ resistance meter with STX-2 electrode to characterize the formation of a tight endothelial cell monolayer. The measurement of a blank filter, i.e. without cells, was performed and the signal was subtracted from that recorded for the filter with cells. Then the resulting value was reported to the membrane area to obtain the TEER measurement in $\Omega . \mathrm{cm}^{2}$.

\subsection{Proteins expression}

\subsubsection{Whole cell ELISA}

HBEC-5i cells were fixed with $4 \%$ paraformaldehyde and permeabilized with $0.1 \%$ Triton-100-X in PBS containing 1\% Bovine Serum Albumin (BSA). Phenotypic marker expression and $\mathrm{ABC}$ transporters proteins were evaluated with primary polyclonal antibodies at the optimal concentration between $2-6 \mu \mathrm{g} / \mathrm{ml}$ for all antibodies. Secondary IgG biotinylated antibody was added. Then, streptavidin was added during 20 min. 3, 3', 5, 5'tetramethylbenzidine (TMB) substrate was added in each well. At the end, the reaction was stopped by addition of $\mathrm{HCl} 1 \mathrm{~N}$. The color reaction was measured with a spectrophotometer (Multiskan ${ }^{\mathrm{TM}} \mathrm{RC}$, Thermo Fisher Scientific, France) at $450 \mathrm{~nm}$. Results were normalized with the total protein content assay, according to the manufacturer's instructions.

\subsubsection{Immunofluorescence assays}

The immunofluorescence method had been previously described (Forest et al., 2015; He et al., 2011). Briefly, inserts were fixed in glacial methanol (pre-stored at $-20^{\circ} \mathrm{C}$ ) for 6 minutes (for $\mathrm{TJ} s$ ) or $1 \%$ PFA (for $\mathrm{ABC}$ transporters) at RT for $30 \mathrm{~min}$. PFA-fixed cells were 
permeabilized with $1 \%$ Triton X-100 for 5 min at RT. The non-specific binding sites were then blocked for $30 \mathrm{~min}$ at $37^{\circ} \mathrm{C}$ in blocking buffer that was $2 \%$ goat serum and $2 \%$ BSA in PBS. The primary antibodies were diluted at $1 / 200$ in the blocking buffer and incubated for 1 hour at $37^{\circ} \mathrm{C}$. The secondary antibodies were diluted at $1 / 500$ in the blocking buffer and incubated for $45 \mathrm{~min}$ at $37^{\circ} \mathrm{C}$. Nuclei were finally counterstained with DAPI diluted $1 / 1000$ in PBS at room temperature for $30 \mathrm{~min}$. The inserts were finally placed on glass slides, submerged with Vectashield Mounting Medium (H1000, Vector Laboratories,USA), and covered with glass coverslip. The staining of all biomarkers had been imaged using an epifluorescence microscope IX81 (Olympus, Tokyo, Japan) equipped with the CellSens imaging software (Olympus, Munster, Germany).

\subsection{ABC transporter functional studies}

\subsubsection{Accumulation studies}

The functionality of the P-gp, MRPs and BCRP ABC transporter was evaluated at the cellular level by targeted accumulation studies based on three fluorescent substrates, BCECF $(2 \mu \mathrm{M})$, rhodamine $123(10 \mu \mathrm{M})$ and Hoechst $33342(20 \mu \mathrm{M})$, as broad spectrum substrate of MRPs and substrates of P-gp and BCRP or selective substrate of BCRP, respectively.

Cells were pre-treated for 15 min at $37^{\circ} \mathrm{C}$ with a range of inhibitors such as verapamil (100 $\mu \mathrm{M})$, probenecid $(100 \mu \mathrm{M})$, cyclosporin $\mathrm{A}(100 \mu \mathrm{M})$ and Ko143 $(10 \mu \mathrm{M})$. The fluorescent probes were then diluted independently in each inhibitor solution prior to further incubation for $1 \mathrm{~h}$ at $37^{\circ} \mathrm{C}$. Finally, the cells were washed with PBS at $4^{\circ} \mathrm{C}$ and lysed at room temperature during $30 \mathrm{~min}$ in a solution of sodium dodecyl sulfate containing $1 \%$ of $10 \mathrm{mM}$ sodium borate. Fluorescence in lysed cells was measured with a fluorescence spectrophotometer with $485 \mathrm{~nm}$ excitation and $530 \mathrm{~nm}$ emission wavelengths for BCECF and rhodamine 123. For Hoechst 33342 , the fluorescence was measured with a fluorescence at $355 / 460 \mathrm{~nm}$. The result was normalized by micro BCA total protein assay according to the manufacturer's instructions. 


\subsubsection{Bidirectional studies and efflux ratio (ER) measurements}

We investigated the permeability of both therapeutic compounds and selective substrates of $\mathrm{ABC}$ transporters using a standardized bi-directional transport protocol according to FDA guidelines (Food and drug administration, 2012). Rhodamine $123(10 \mu \mathrm{M})$ was evaluated as a P-gp and BCRP substrate with verapamil and Ko143 as selective inhibitors. BCECF at $10 \mu \mathrm{M}$ was assessed as a broad spectrum MRPs substrate with probenecid as a general MRPs inhibitor. Rivaroxaban $(10 \mu \mathrm{M})$ was evaluated as P-gp and BCRP substrate with verapamil and Ko143. Methotrexate $(100 \mu \mathrm{M})$ was assessed as a MRP and BCRP substrate with probenecid as a general MRPs inhibitor and Ko143 as inhibitor of BCRP.

The experiment consisted of two mono-directional assays of trans-endothelial passage, with the tested drug initially added either in the apical compartment to obtain the apical to basolateral permeability $\left(\mathrm{P}_{\mathrm{app} \mathrm{A} \rightarrow \mathrm{B}}\right)$ or in the basolateral compartment to obtain the basolateralto-apical permeability $\left(\mathrm{P}_{\text {app }} \mathrm{B} \rightarrow \mathrm{A}\right)$ with the Formula 1

To determine whether the tested compounds underwent active efflux by ABCs transporters, the ER of each compound was computed according to the following formula:

$$
\mathrm{ER}=P_{a p p}(B \rightarrow A) / P_{a p p}(A \rightarrow B) \text { Formula } 2
$$

The standard deviation of the ER being calculated according to the formula proposed by Gnoth et al. (Gnoth et al., 2011). According to FDA guidelines an ER $>2$ indicates that the drug is an efflux transporter substrate. This ER must be decreased with selective inhibitor. The inhibitor must halve of the ER or make it less than 2 (Food and drug administration, 2012).

Percent of ER inhibition was computed using the Formula 3 (Hodin et al., 2017):

$$
\text { ER inhibition }(\%)=1-\frac{E R \text { inhibitor }}{E R} \times 100 \text { Formula } 3
$$

\subsubsection{Liquid chromatography - mass spectrometry analysis}


Rivaroxaban, methotrexate and caffeine were quantified using an Aquity UPLC system coupled with a Xevo TQ-S triple quadrupole mass spectrometer (Waters, Saint-Quentin-enYvelines, France). Positive ionization condition was used for analysis of rivaroxaban $(\mathrm{m} / \mathrm{z}$ $436.07 \rightarrow 231.11)$, methotrexate $(\mathrm{m} / \mathrm{z} 455.12 \rightarrow 308.17)$, and caffeine $(\mathrm{m} / \mathrm{z} 195.09 \rightarrow 138.01)$. The internal standard (IS) for each drug were $\left[{ }^{13} \mathrm{C}_{6}\right]$-rivaroxaban $(\mathrm{m} / \mathrm{z} 442.09 \rightarrow 144.95),\left[{ }^{13} \mathrm{C}\right.$, ${ }^{2} \mathrm{H}_{3}$ ]-methotrexate $(\mathrm{m} / \mathrm{z} 458.12 \rightarrow 311.17),\left[{ }^{3} \mathrm{H}_{3}\right]$-caffeine $(\mathrm{m} / \mathrm{z} 204.16 \rightarrow 144.1)$ respectively. Samples $(50 \mu \mathrm{L})$ were extracted with $500 \mu \mathrm{L}$ of IS in methanol. The mobile phase comprised a mixture of water containing $0.1 \%$ formic acid (FA) and acetonitrile containing $0.1 \%$ FA. An eluate gradient was applied to a BEH C18 column $(50 \mathrm{~mm} \times 2.1 \mathrm{~mm} \times 2.7 \mu \mathrm{m})$ (Waters, SaintQuentin-en-Yvelines, France). For the permeability calculation, the peak area ratios of the drugs and their respective IS were used as $\mathrm{C} 0$ and $\mathrm{C} 1$.

\subsection{Statistical Analysis}

Graphpad software was used for data analysis and graphic outputs. Data were expressed as the mean \pm standard deviation. Statistical analyses were performed using Graphpad software (Graphpad software Incorporation, San Diego, USA). A Mann-Whiney test was used. Treated samples were compared to untreated control. The differences were considered statistically significant when p-value $<0.05$.

\section{Results}

\subsection{Development and characterization of the model}

To develop the model, the first step was to adapt endothelial and astrocytic cells in the same culture medium without adjuvant. The expression of two phenotypic markers, GFAP (Glial Fibrillary Acidic Protein) for astrocytes and VE-cadherin for endothelial cells were performed and were stable along the culture experiments (data not shown). 
In a second step, we compared two endothelial cells monoculture conditions: a standard culture medium (HBEC-5i alone) and astrocytes conditioned medium (HBEC-5i with HA conditioned medium). TEER gradually increased to reach a steady state plateau at day 19 which was maintained during two days for HBEC-5i alone. The steady state plateau was reached at day 14 and maintained during five days for HBEC-5i with HA conditioned medium (Table 1).

In parallel, permeability studies were realized with two molecules: Na-Fl (376 Da) and dextran-FITC (4 kDa). The lowest $\mathrm{P}_{\text {app }}$ values were obtained for the HBEC-5i with HA conditioned medium (Table 1). The $\mathrm{P}_{\text {app }}$ value for the dextran-FITC was lower than the $\mathrm{P}_{\text {app }}$ value for Na-Fl, in agreement with the molecular weight of the compounds. All these results indicated that HBEC-5i with HA conditioned medium allowed to have a higher tightness of the barrier compared to the HBEC-5i alone.

HBEC5i cells were able to form a monolayer on insert and to express TJs proteins: ZO1 and claudin-5 as represented in figure $1 \mathrm{AB}$.

\subsection{Expression of $\mathrm{ABC}$ transporters}

The expression of $\mathrm{ABC}$ transporters was evaluated with semi-quantitative protein expression by whole cell ELISA assay. We verified whether, astrocytes conditioned medium was able to induce any modification of the $\mathrm{ABC}$ transporters expression (figure $2 \mathrm{ABCD}$ ). $\mathrm{P}$ gp, MRP-1, MRP-2 and BCRP had the same expression in the two culture media. In this experiment, we also used cells cultivated during 7 days with hydrocortisone as a positive control, as reported in the literature to obtain a tight model (Kulczar et al., 2017). The hydrocortisone increased P-gp expression 1.8 fold and BCRP expression 1.3 fold on our HBEC5i model. On the contrary the MRPs expression was not modified by hydrocortisone treatment. These results support the idea that adjuvants have to be avoided to study drug interactions with $\mathrm{ABC}$ transporter as the main adjuvant reported in the literature is hydrocortisone. 
The expression of P-gp, BCRP, MRP-1 and MRP-2 were also assessed in monolayer inserts of HBEC-5i with immunostaining (figure 2EFGH).

\subsection{Functionality of $\mathrm{ABC}$ transporters}

\subsubsection{Accumulation studies}

The functionality of the $\mathrm{ABC}$ transporters was assessed at the cellular level in standard and conditioned media conditions by means of accumulation assays. These experiments were based on the use of three fluorescent substrates BCECF (MRPs), rhodamine 123 (P-gp) and Hoechst 33342 (BCRP) incubated with or without inhibitors: verapamil, probenecid, Ko143 against P-gp, MRPs and BCRP respectively. In addition, cyclosporin A, a large spectrum inhibitor has been used (figure $3 \mathrm{ABC}$ ). In all experiments, we compared the two culture conditions.

The functionality of ABC transporters on HBEC-5i endothelial cells was similar when cells were cultured either in standard medium or in astrocytes medium. The addition of cyclosporine A induced an increase of the accumulation of all fluorescent substrates, which is in agreement with the large spectrum inhibition of cyclosporin A. For example, the addition of cyclosporin A induced an accumulation of Hoechst 33342 three-fold higher than in control cells, whatever the culture condition (figure 3A).

The dual use of substrates and inhibitors allowed to demonstrate the P-gp, MRPs and BCRP transporters functionalities in our model. For instance, the accumulation of BCECF in cells treated with probenecid increased to $286 \pm 34 \%$ for the standard medium and $276 \pm 24 \%$ for the conditioned medium (figure 3C).

\subsubsection{Bidirectional permeability studies}

Bidirectional permeability measurement that lead to ER allow to complete the evaluation of the ABC transporters functionality in a barrier model. The Hoechst fluorophore could not be used 
for bidirectional studies because this fluorescent molecule can be trapped in the nucleus to stain DNA of endothelial cells.

We noticed that the presence of HA conditioned medium allowed a better functionality of ABC transporters with higher ER (figure 4).

The ER of rhodamine 123 was 2.3 fold higher in the HBEC-5i cultured in the presence of HA conditioned medium than in HBEC-5i cultured alone. In the HA conditioned medium, the use of Ko143 and verapamil decreased ER value by $49 \%$ and by $65 \%$ respectively (figure $4 \mathrm{AB})$.

The ER of BCECF was 2.8 fold higher in HBEC-5i with the HA conditioned medium than in HBEC-5i alone. Inhibition with probenecid allowed a decrease of $52 \%$ in HBEC-5i with the HA conditioned medium (Figure $4 \mathrm{C}$ ).

Taken together, these results indicate that in our model, ABC transporters are functional. In a last part, and as reported by figure 5, we investigated the permeability profile of three pharmaceutical agents: rivaroxaban, methotrexate and caffeine in HBEC-5i with the HA conditioned medium.

Caffeine is a hydrophilic molecule, which crosses the barrier by a para-cellular pathway. We found a permeability value of $67.0 \pm 4.4 \times 10^{-6} \mathrm{~cm} \cdot \mathrm{s}^{-1}$. This value is higher than the value for the Na-Fl and the dextran-FITC (figure 5A). These results are in agreement with the lowest molecular weight of this molecule compared to the others.

We evaluated the bidirectional transport of two drugs known to be substrates of $\mathrm{ABC}$ transporters. The rivaroxaban was released outside the cells forming the barrier with an efflux ratio at $10.1 \pm 1.2$ (figure 5B). The use of verapamil and Ko143 drastically inhibited the efflux of rivaroxaban by $79 \%$ and $91 \%$ respectively. These results indicated that in our model, rivaroxaban is transported by $\mathrm{BCRP}$ and $\mathrm{P}$-gp. 
The methotrexate was released outside cells with an efflux ratio at $3.1 \pm 0.2$ (figure 5C). The Ko143 and the probenecid diminished the ER below 2. The use of probenecid and Ko143 inhibited the efflux of rivaroxaban by $59 \%$ and $69 \%$ respectively. These results confirmed that in our model, methotrexate is transported by MRPs and BCRP. These results show that in our model $\mathrm{ABC}$ transporters can transport pharmaceutical molecules.

\section{Discussion}

The transport of drugs from the blood to the brain mainly depends on their properties and interaction with $\mathrm{ABC}$ transporters (Bernacki et al., 2008). To study the $\mathrm{ABC}$ transporters at the $\mathrm{BBB}$, it is necessary to develop a suitable barrier model based on brain endothelial cells grown as monoculture on inserts (Puech et al., 2018).

To that purpose, we developed a model composed of HBEC-5i cells cultivated either in standard medium or in the presence of HA conditioned medium. We first characterized the tightness of our model. When HBEC-5i cells were cultured with the HA conditioned medium it took 14 days to the model to reach stability (maximal TEER) which was maintained for 5 days. In comparison, the model cultivated with standard medium allowed the maximal TEER value after19 days and was stable only during 2 days. Similarly, the other models of human BBB found in the literature, show a short stability, about 2 days only (Biemans et al., 2017; Eigenmann et al., 2016b, 2013; Weksler et al., 2013). We also observed that HBEC-5i cells with HA conditioned medium exhibited a better permeability for Na-Fl than the hCMEC/D3 cells commonly used in the literature $\left(5.7 \times 10^{-6} \mathrm{~cm} \cdot \mathrm{s}^{-1}\right.$ and $13 \times 10^{-6} \mathrm{~cm} \cdot \mathrm{s}^{-1}$ respectively) (Eigenmann et al., 2013). HBEC-5i cells expressed ZO-1 and claudin-5 the two essential TJs involved as reported in the literature with hCMEC/D3 (Eigenmann et al., 2013; Kulczar et al., 2017; Poller et al., 2008; Weksler et al., 2005). We highlighted with immunofluorescent staining that our model exhibited the same characteristic membrane distribution of TJs for brain vascular endothelial cells as hCMEC/D3 (Adriani et al., 2017; Sajja et al., 2014). The 
expression of tight junctions in our model limited the passage of small molecules in the paracellular pathways, it was correlated with the result of the $\mathrm{P}_{\text {app }}$ for dextran-FITC and Na-Fl. The results obtained showed that astrocytes cooperate with brain endothelial cells and are necessary in the acquisition of a better tightness of a BBB (Abbott et al., 2006; Dehouck et al., 1990; Kulczar et al., 2017). Most of co-culture models found in the literature allow to observe the essential role of astrocytes on the tightness and permeability, i.e. the physical barrier properties (Janzer and Raff, 1987; Neuhaus et al., 2014). More precisely, it has been shown that the cocultures with astrocytes induce a higher tightness of the BBB composed either of the HCMEC/D3 or HBMEC cell lines (Hatherell et al., 2011; Kulczar et al., 2017; Kuo and Lu, 2011).

In a second part, we demonstrated that our HBEC-5i model expressed P-gp, MRP1, 2 and BCRP which are the most representative human in vivo ABC transporters (Abbott, 2013). Our experiments with hydrocortisone reinforced the need to not use this compound in culture medium as previously discussed (Eisenblätter and Galla, 2002; Maines et al., 2005; von WedelParlow et al., 2009). We have also demonstrated the functionality of ABC transporters with accumulation studies with specific substrates as described in the literature (Bachmeier et al., 2004; Kodama et al., 2016; Poller et al., 2008). As expected, we showed that all the ABC transporters in our model were present and functional as for hCMEC/D3 and HBMEC (Kuo and Lu, 2012; Weksler et al., 2013).

In a third part, we performed bilateral studies, to determine ER in our model. This parameter completely determines the interaction between the drugs and $\mathrm{ABC}$ transporter function (Food and drug administration, 2012; Hubatsch et al., 2007; Kikuchi et al., 2013). To date, only few studies on human BBB cell lines have evaluated this parameter (Eigenmann et al., 2016a; Jähne et al., 2016; Ma et al., 2014). In our model, ER parameter was higher in HBEC$5 \mathrm{i}$ with the HA conditioned medium than HBEC-5i alone. This result is the first to demonstrate 
on a BBB model that astrocyte cooperation is necessary in $\mathrm{ABC}$ transporters bilateral studies. There are only few studies on the effect of the astrocytes on metabolic properties of the BBB involving $\mathrm{ABC}$ transporters expression. In hCMEC/D3 a contact co-culture model with human astrocytes, has shown a lower accumulation in rhodamine 123, a fluorescent substrate of P-gp, suggesting that the level of this $\mathrm{ABC}$ transporter is higher compared to the mono-culture condition (Kulczar et al., 2017).

The co-culture contact model prevents the bilateral studies, because there are two monolayers on both sides of the insert (Puech et al., 2018). This configuration can induce an under or overestimation of the involvement of the ABC transporters in the drug transport studies. The noncontact co-culture configuration can be an alternative to the drawback of the co-culture contact, with the assessment of ER on a monolayer of endothelial cells (Eigenmann et al., 2016a).

In a last part, we evaluated the capacity of our model to discriminate pharmacological molecules in agreement with their properties. The result obtained for the caffeine is in agreement with the result obtained in the literature in the HBMEC cell line (Eigenmann et al., 2016b). In our model and in accordance with the literature the rivaroxaban was transported by BRCP and P-gp (Gnoth et al., 2011; Hodin et al., 2017). The value of the ER for methotrexate was in agreement with the presence of MRPs at the basolateral and apical membrane of the endothelial cells. Methotrexate was transported by MRPs, its active efflux by one MRP transporter in the apical compartment could be balanced by the active efflux in the basolateral compartment by other MRPs (Wolf et al., 2005). We have clearly demonstrated that our BBB model can evaluate interaction or not with $\mathrm{ABC}$ transporters.

To conclude, we have developed a suitable human BBB model composed of endothelial cell line HBEC-5i. This model offers several advantages like easy to use and can be used routinely in a laboratory to screen drug interactions. In addition, our model presents many advantages which are not found on the other BBB models available in the literature. For 
instance, a stability during five days which may allow to screen long term effects of therapeutic agents. Furthermore, the culture is performed without adjuvant and gives the possibility to realize ER with pharmacological molecules. Our model can be used to predict the permeability of therapeutic molecules across the BBB.

\section{Conflict of interest statement}

The authors declare that they have no conflict of interest.

\section{References:}

Abbott, N.J., 2013. Blood-brain barrier structure and function and the challenges for CNS drug delivery. J. Inherit. Metab. Dis. 36, 437-449. https://doi.org/10.1007/s10545-013-9608-0

Abbott, N.J., Rönnbäck, L., Hansson, E., 2006. Astrocyte-endothelial interactions at the blood-brain barrier. Nat. Rev. Neurosci. 7, 41-53. https://doi.org/10.1038/nrn1824

Ades, E.W., Candal, F.J., Swerlick, R.A., George, V.G., Summers, S., Bosse, D.C., Lawley, T.J., 1992. HMEC-1: establishment of an immortalized human microvascular endothelial cell line. J. Invest. Dermatol. 99, 683-690.

Adriani, G., Ma, D., Pavesi, A., Kamm, R.D., Goh, E.L.K., 2017. A 3D neurovascular microfluidic model consisting of neurons, astrocytes and cerebral endothelial cells as a blood-brain barrier. Lab. Chip 17, 448-459. https://doi.org/10.1039/c6lc00638h

Bachmeier, C.J., Trickler, W.J., Miller, D.W., 2004. Drug Efflux Transport Properties of 2',7'-Bis(2carboxyethyl)-5(6)-carboxyfluorescein Acetoxymethyl Ester (BCECF-AM) and Its Fluorescent Free Acid, BCECF. J. Pharm. Sci. 93, 932-942. https://doi.org/10.1002/jps.20018

Berezowski, V., Fukuda, A.M., Cecchelli, R., Badaut, J., 2012. Endothelial Cells and Astrocytes: A Concerto en Duo in Ischemic Pathophysiology. Int. J. Cell Biol. 2012. https://doi.org/10.1155/2012/176287

Bernacki, J., Dobrowolska, A., Nierwińska, K., Małecki, A., 2008. Physiology and pharmacological role of the blood-brain barrier. Pharmacol. Rep. PR 60, 600-622.

Biemans, E.A.L.M., Jäkel, L., de Waal, R.M.W., Kuiperij, H.B., Verbeek, M.M., 2017. Limitations of the hCMEC/D3 cell line as a model for $\mathrm{A} \beta$ clearance by the human blood-brain barrier. J. Neurosci. Res. 95, 1513-1522. https://doi.org/10.1002/jnr.23964

Dehouck, M.P., Méresse, S., Delorme, P., Fruchart, J.C., Cecchelli, R., 1990. An easier, reproducible, and mass-production method to study the blood-brain barrier in vitro. J. Neurochem. 54, $1798-1801$.

Dohgu, S., Takata, F., Yamauchi, A., Nakagawa, S., Egawa, T., Naito, M., Tsuruo, T., Sawada, Y., Niwa, M., Kataoka, Y., 2005. Brain pericytes contribute to the induction and up-regulation of blood-brain barrier functions through transforming growth factor-beta production. Brain Res. 1038, 208-215. https://doi.org/10.1016/j.brainres.2005.01.027

Eigenmann, D.E., Dürig, C., Jähne, E.A., Smieško, M., Culot, M., Gosselet, F., Cecchelli, R., Helms, H.C.C., Brodin, B., Wimmer, L., Mihovilovic, M.D., Hamburger, M., Oufir, M., 2016a. In vitro blood-brain barrier permeability predictions for GABAA receptor modulating piperine analogs. Eur. J. Pharm. Biopharm. Off. J. Arbeitsgemeinschaft Pharm. Verfahrenstechnik EV 103, 118-126. https://doi.org/10.1016/j.ejpb.2016.03.029

Eigenmann, D.E., Jähne, E.A., Smieško, M., Hamburger, M., Oufir, M., 2016b. Validation of an immortalized human (hBMEC) in vitro blood-brain barrier model. Anal. Bioanal. Chem. 408, 2095-2107. https://doi.org/10.1007/s00216-016-9313-6 
Eigenmann, D.E., Xue, G., Kim, K.S., Moses, A.V., Hamburger, M., Oufir, M., 2013. Comparative study of four immortalized human brain capillary endothelial cell lines, hCMEC/D3, hBMEC, TY10, and BB19, and optimization of culture conditions, for an in vitro blood-brain barrier model for drug permeability studies. Fluids Barriers CNS 10, 33. https://doi.org/10.1186/2045-8118-10-33

Eisenblätter, T., Galla, H.-J., 2002. A new multidrug resistance protein at the blood-brain barrier. Biochem. Biophys. Res. Commun. 293, 1273-1278. https://doi.org/10.1016/S0006291X(02)00376-5

Food and drug administration, 2012. Guidance for industry drug interaction studies. https://www.fda.gov/downloads/drugs/ guidances/ucm292362.pdf. Assessed 18 may 2018.

Forest, F., Thuret, G., Gain, P., Dumollard, J.-M., Peoc'h, M., Perrache, C., He, Z., 2015. Optimization of immunostaining on flat-mounted human corneas. Mol. Vis. 21, 1345-1356.

Gnoth, M.J., Buetehorn, U., Muenster, U., Schwarz, T., Sandmann, S., 2011. In vitro and in vivo Pglycoprotein transport characteristics of rivaroxaban. J. Pharmacol. Exp. Ther. 338, 372-380. https://doi.org/10.1124/jpet.111.180240

Hatherell, K., Couraud, P.-O., Romero, I.A., Weksler, B., Pilkington, G.J., 2011. Development of a three-dimensional, all-human in vitro model of the blood-brain barrier using mono-, co-, and tri-cultivation Transwell models. J. Neurosci. Methods 199, 223-229. https://doi.org/10.1016/j.jneumeth.2011.05.012

He, Z., Campolmi, N., Ha Thi, B.-M., Dumollard, J.-M., Peoc'h, M., Garraud, O., Piselli, S., Gain, P., Thuret, G., 2011. Optimization of immunolocalization of cell cycle proteins in human corneal endothelial cells. Mol. Vis. 17, 3494-3511.

Helms, H.C., Abbott, N.J., Burek, M., Cecchelli, R., Couraud, P.-O., Deli, M.A., Förster, C., Galla, H.J., Romero, I.A., Shusta, E.V., Stebbins, M.J., Vandenhaute, E., Weksler, B., Brodin, B., 2016. In vitro models of the blood-brain barrier: An overview of commonly used brain endothelial cell culture models and guidelines for their use. J. Cereb. Blood Flow Metab. 36, 862-890. https://doi.org/10.1177/0271678X16630991

Hodin, S., Basset, T., Jacqueroux, E., Delezay, O., Clotagatide, A., Perek, N., Mismetti, P., Delavenne, X., 2017. In Vitro Comparison of the Role of P-Glycoprotein and Breast Cancer Resistance Protein on Direct Oral Anticoagulants Disposition. Eur. J. Drug Metab. Pharmacokinet. https://doi.org/10.1007/s13318-017-0434-x

Hubatsch, I., Ragnarsson, E.G.E., Artursson, P., 2007. Determination of drug permeability and prediction of drug absorption in Caco-2 monolayers. Nat. Protoc. 2, 2111-2119. https://doi.org/10.1038/nprot.2007.303

Jähne, E.A., Eigenmann, D.E., Sampath, C., Butterweck, V., Culot, M., Cecchelli, R., Gosselet, F., Walter, F.R., Deli, M.A., Smieško, M., Hamburger, M., Oufir, M., 2016. Pharmacokinetics and In Vitro Blood-Brain Barrier Screening of the Plant-Derived Alkaloid Tryptanthrin. Planta Med. 82, 1021-1029. https://doi.org/10.1055/s-0042-105295

Janzer, R.C., Raff, M.C., 1987. Astrocytes induce blood-brain barrier properties in endothelial cells. Nature 325, 253-257. https://doi.org/10.1038/325253a0

Jiang, W., Huang, W., Chen, Y., Zou, M., Peng, D., Chen, D., 2017. HIV-1 Transactivator Protein Induces ZO-1 and Neprilysin Dysfunction in Brain Endothelial Cells via the Ras Signaling Pathway. Oxid. Med. Cell. Longev. 2017. https://doi.org/10.1155/2017/3160360

Kikuchi, R., de Morais, S.M., Kalvass, J.C., 2013. In vitro P-glycoprotein efflux ratio can predict the in vivo brain penetration regardless of biopharmaceutics drug disposition classification system class. Drug Metab. Dispos. Biol. Fate Chem. 41, 2012-2017. https://doi.org/10.1124/dmd.113.053868

Kodama, N., Iwao, T., Katano, T., Ohta, K., Yuasa, H., Matsunaga, T., 2016. Characteristic Analysis of Intestinal Transport in Enterocyte-Like Cells Differentiated from Human Induced Pluripotent Stem Cells. Drug Metab. Dispos. Biol. Fate Chem. 44, 0. https://doi.org/10.1124/dmd.116.069336

Kulczar, C., Lubin, K.E., Lefebvre, S., Miller, D.W., Knipp, G.T., 2017. Development of a direct contact astrocyte-human cerebral microvessel endothelial cells blood-brain barrier coculture model. J. Pharm. Pharmacol. 69, 1684-1696. https://doi.org/10.1111/jphp.12803 
Kuo, Y.-C., Lu, C.-H., 2012. Expression of P-glycoprotein and multidrug resistance-associated protein on human brain-microvascular endothelial cells with electromagnetic stimulation. Colloids Surf. B Biointerfaces 91, 57-62. https://doi.org/10.1016/j.colsurfb.2011.10.035

Kuo, Y.-C., Lu, C.-H., 2011. Effect of human astrocytes on the characteristics of human brainmicrovascular endothelial cells in the blood-brain barrier. Colloids Surf. B Biointerfaces 86, 225-231. https://doi.org/10.1016/j.colsurfb.2011.04.005

Ma, S., Liu, X., Xu, Q., Zhang, X., 2014. Transport of ginkgolides with different lipophilicities based on an hCMEC/D3 cell monolayer as a blood-brain barrier cell model. Life Sci. 114, 93-101. https://doi.org/10.1016/j.lfs.2014.08.006

Maines, L.W., Antonetti, D.A., Wolpert, E.B., Smith, C.D., 2005. Evaluation of the role of Pglycoprotein in the uptake of paroxetine, clozapine, phenytoin and carbamazapine by bovine retinal endothelial cells. Neuropharmacology 49, 610-617. https://doi.org/10.1016/j.neuropharm.2005.04.028

Muruganandam, A., Herx, L.M., Monette, R., Durkin, J.P., Stanimirovic, D.B., 1997. Development of immortalized human cerebromicrovascular endothelial cell line as an in vitro model of the human blood-brain barrier. FASEB J. Off. Publ. Fed. Am. Soc. Exp. Biol. 11, 1187-1197.

Neuhaus, W., Gaiser, F., Mahringer, A., Franz, J., Riethmüller, C., Förster, C., 2014. The pivotal role of astrocytes in an in vitro stroke model of the blood-brain barrier. Front. Cell. Neurosci. 8, 352. https://doi.org/10.3389/fncel.2014.00352

Pardridge, W.M., 2005. The Blood-Brain Barrier: Bottleneck in Brain Drug Development. NeuroRX, The Blood-Brain Barrier and Neurotherapeutics 2, 3-14. https://doi.org/10.1602/neurorx.2.1.3

Poller, B., Gutmann, H., Krähenbühl, S., Weksler, B., Romero, I., Couraud, P.-O., Tuffin, G., Drewe, J., Huwyler, J., 2008. The human brain endothelial cell line hCMEC/D3 as a human bloodbrain barrier model for drug transport studies. J. Neurochem. 107, 1358-1368.

Prudhomme, J.G., Sherman, I.W., Land, K.M., Moses, A.V., Stenglein, S., Nelson, J.A., 1996. Studies of Plasmodium falciparum cytoadherence using immortalized human brain capillary endothelial cells. Int. J. Parasitol. 26, 647-655.

Puech, C., Delavenne, X., Perek, N., 2018. The expected characteristics of an in vitro human Blood Brain Barrier model derived from cell lines, for studying how ABC transporters influence drug permeability. J. Drug Deliv. Sci. Technol. 45, 159-167. https://doi.org/10.1016/j.jddst.2018.03.002

Ronaldson, P.T., Davis, T.P., 2015. Targeting transporters: promoting blood-brain barrier repair in response to oxidative stress injury. Brain Res. 1623, 39-52. https://doi.org/10.1016/j.brainres.2015.03.018

Sajja, R., Prasad, S., Cucullo, L., 2014. Impact of altered glycaemia on blood-brain barrier endothelium: An in vitro study using the hCMEC/D3 cell line. Fluids Barriers CNS 11, 8. https://doi.org/10.1186/2045-8118-11-8

Sano, Y., Shimizu, F., Abe, M., Maeda, T., Kashiwamura, Y., Ohtsuki, S., Terasaki, T., Obinata, M., Kajiwara, K., Fujii, M., Suzuki, M., Kanda, T., 2010. Establishment of a new conditionally immortalized human brain microvascular endothelial cell line retaining an in vivo blood-brain barrier function. J. Cell. Physiol. 225, 519-528. https://doi.org/10.1002/jcp.22232

von Wedel-Parlow, M., Wölte, P., Galla, H.-J., 2009. Regulation of major efflux transporters under inflammatory conditions at the blood-brain barrier in vitro. J. Neurochem. 111, 111-118. https://doi.org/10.1111/j.1471-4159.2009.06305.x

Weksler, B., Romero, I.A., Couraud, P.-O., 2013. The hCMEC/D3 cell line as a model of the human blood brain barrier. Fluids Barriers CNS 10, 16. https://doi.org/10.1186/2045-8118-10-16

Weksler, B.B., Subileau, E.A., Perrière, N., Charneau, P., Holloway, K., Leveque, M., TricoireLeignel, H., Nicotra, A., Bourdoulous, S., Turowski, P., Male, D.K., Roux, F., Greenwood, J., Romero, I.A., Couraud, P.O., 2005. Blood-brain barrier-specific properties of a human adult brain endothelial cell line. FASEB J. Off. Publ. Fed. Am. Soc. Exp. Biol. 19, 1872-1874. https://doi.org/10.1096/fj.04-3458fje

Wolf, J., Stranzl, T., Filipits, M., Pohl, G., Pirker, R., Leeb, B., Smolen, J., 2005. Expression of resistance markers to methotrexate predicts clinical improvement in patients with rheumatoid arthritis. Ann. Rheum. Dis. 64, 564-568. https://doi.org/10.1136/ard.2003.014985 


\begin{tabular}{|c|c|c|c|c|c|}
\hline \multirow{2}{*}{ Conditions } & \multicolumn{3}{|c|}{ TEER } & \multicolumn{2}{c|}{ Papp mean max $\left({\left.\mathrm{cm} . \mathrm{s}^{-1}\right)}^{-}\right.$} \\
\cline { 2 - 6 } & Mean max $\Omega . \mathrm{cm}^{2}$ & Days & Stability & Na-FI & Dextran-FITC \\
\hline $\begin{array}{c}\text { HBEC-5i } \\
\text { alone }\end{array}$ & $35.8 \pm 2.14$ & 19 & 2 days & $7.8 \pm 0.1 \times 10^{-6}$ & $6.4 \pm 0.2 \times 10^{-6}$ \\
\hline $\begin{array}{c}\text { HBEC-5i HA } \\
\text { conditioned } \\
\text { medium }\end{array}$ & $39.8 \pm 0.81$ & 14 & 5 days & $5.7 \pm 0.1 \times 10^{-6}$ & $3.6 \pm 0.1 \times 10^{-6}$ \\
\hline
\end{tabular}

Table 1: Main characteristics of tightness of our model. We have tested two configurations: the HBEC-5i alone and HBEC-5i + HA conditioned medium. Mean TEER and apparent permeability for the Na-Fl and Dextran-FITC 4kDa values over time for human endothelial cell line HBEC-5i on 24 well tissue culture inserts are reported. "Days" corresponds to the time necessary to obtain mean max TEER, and "stability" corresponds to the number of the days during which mean max TEER was maintained. Results are expressed as mean $\pm \mathrm{SD}, \mathrm{n}=3, \mathrm{~N}=3$.

Figure 1: Immunofluorescent staining of ZO-1 (A) and Claudin-5 (B) tight junction proteins. Tight junctions proteins are stained in green, nuclei in blue (DAPI). Scale bar $=50 \mu \mathrm{m}(\mathrm{AB})$.

Figure 2: Protein expression of the BRCP (A), P-gp (B), MRP-1 (C), MRP2 (D) by Whole Cell ELISA in different conditions: HBEC-5i alone, HBEC-5i with HA conditioned medium medium (+ HA conditioned medium) or with hydrocortisone. Results are expressed as mean \pm $\mathrm{SD}, \mathrm{n}=6{ }^{* * *} \mathrm{p}$-value $<0.005$ compared to standard medium as determined by a Mann-Whitney test. Immunofluorescence staining of BCRP (E), P-gp (F), MRP-1 (G), MRP-2 (F) in the HBEC-5i cell line. Scale bar $=50 \mu \mathrm{m}$.

Figure 3: Intracellular accumulation of Hoescht 33342 (A), rhodamine 123 (B) and BCECF (C) inside HBEC-5i cells treated with verapamil, probenecid, Ko143 and cyclosporin A after 60 min of incubation. Two models were evaluated: HBEC-5i alone and HBEC-5i with HA conditioned medium. $\mathrm{N}=6$ for each condition. Intracellular accumulation was normalized to the whole cell content. Data are expressed as the mean $\pm \mathrm{SD}, \mathrm{n}=6$. $* * \mathrm{p}$-value $<0.01$; ***pvalue $<0.005$ compared to untreated control as determined by a Mann-Whitney test.

Figure 4: Bidirectional permeability of rhodamine 123 (Rhodamine) (AB) and BCECF (C) through the HBEC-5i model after 1 hour in HBEC-5i alone or HBEC-5i with HA conditioned medium medium (HA CM). Selective inhibitors used were: Ko143, verapamil and probenecid. Results are expressed as mean $\pm \mathrm{SD}, \mathrm{n}=3$. 
Figure 5: Evaluation of permeability of pharmacological molecules through the HBEC-5i model. Para-cellular permeability of caffeine and fluorescent small molecules (A). Bidirectional permeability of rivaroxaban (riva) (B) and methotrexate $(\mathrm{Mtx})(\mathrm{C})$ through the HBEC-5i model after 1 hour. Selective inhibitors used were: Ko143, verapamil and probenecid. Results are expressed as mean $\pm \mathrm{SD}, \mathrm{n}=3$ for each experiment.

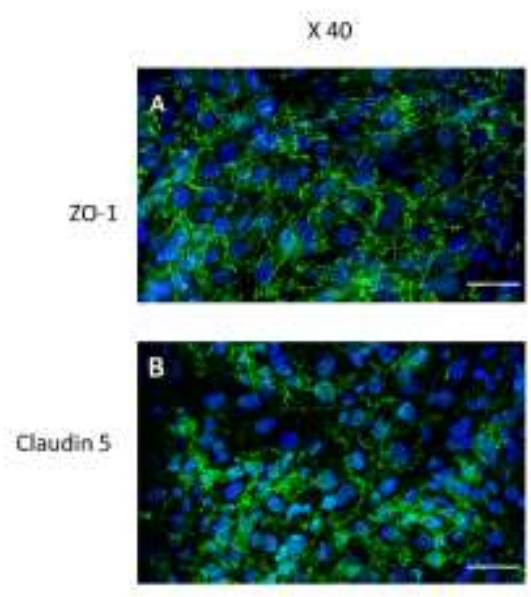



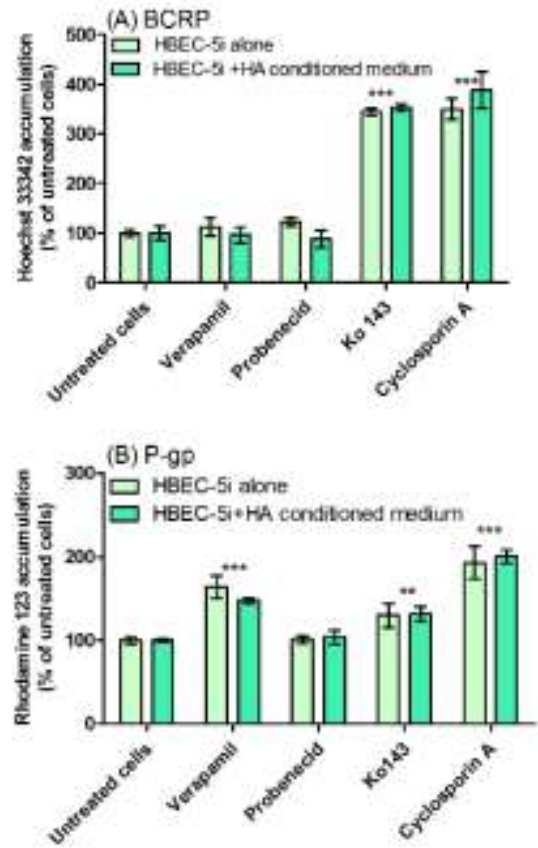

(C) MRPs

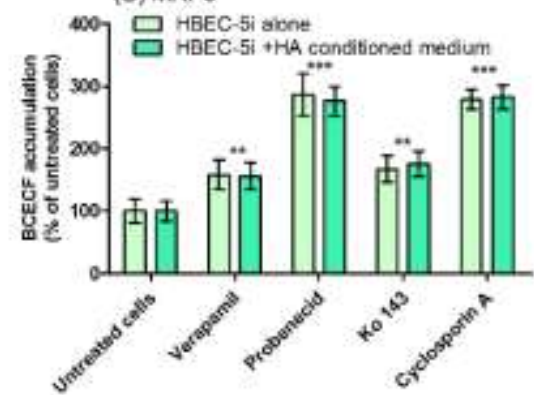



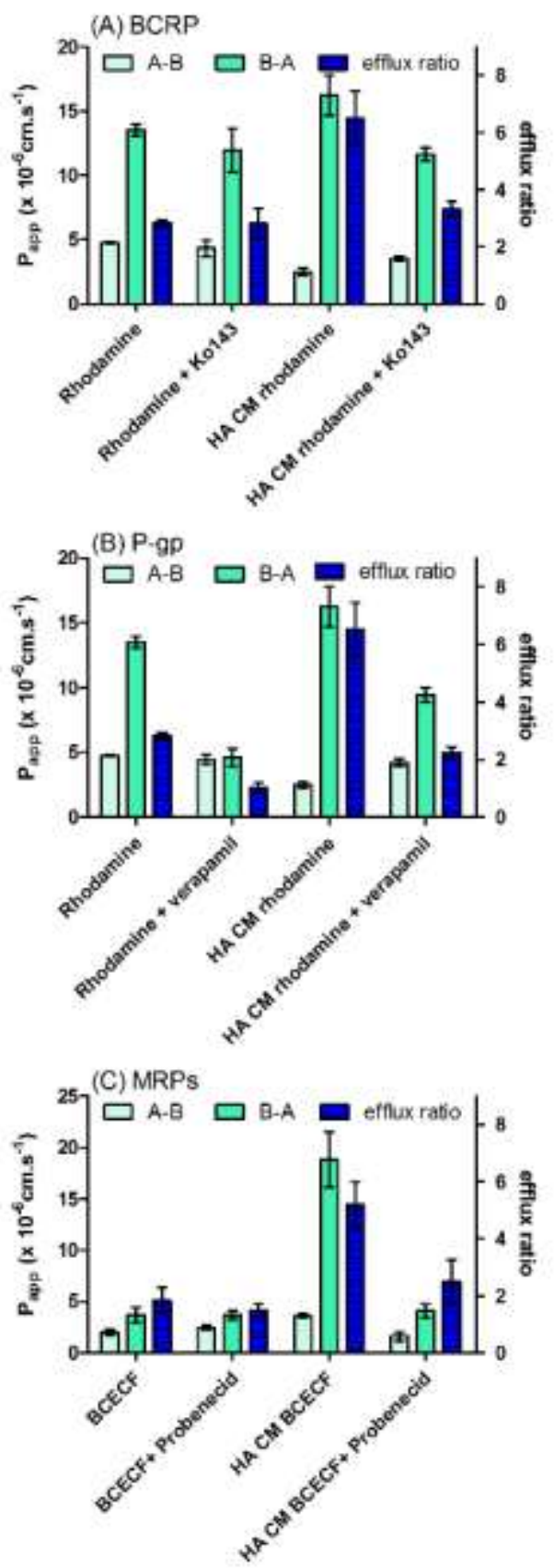

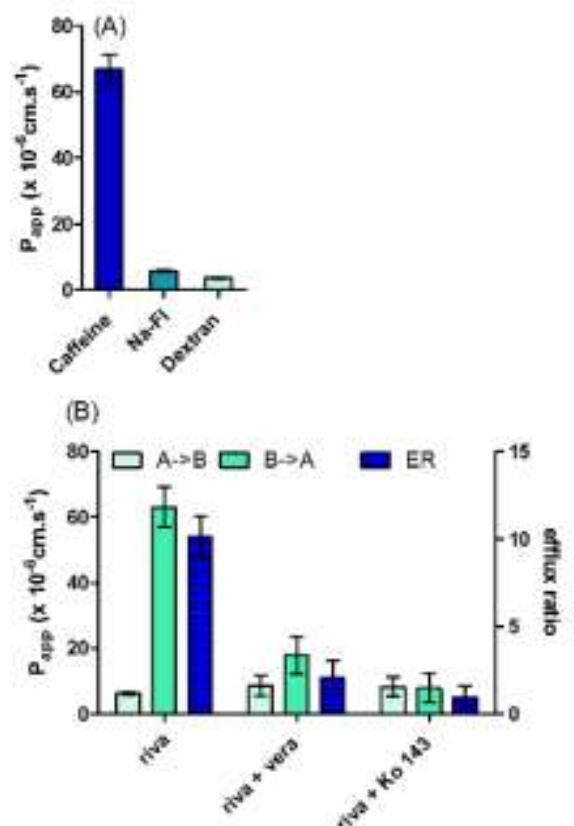

(C)

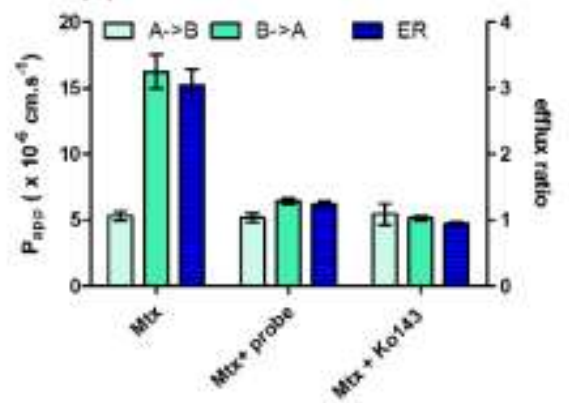

